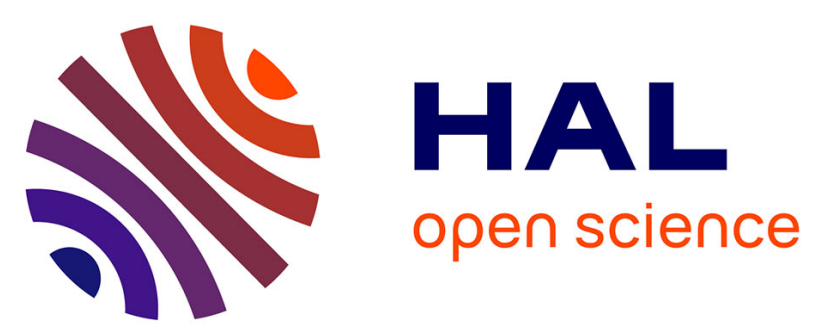

\title{
A Simulation-based Approach to Generate Walking Gait Accelerations for Pedestrian Navigation Solutions
}

\author{
Mahdi Abid, Valérie Renaudin, Thomas Robert, Yannick Aoustin, Eric Le
}

Carpentier

\section{- To cite this version:}

Mahdi Abid, Valérie Renaudin, Thomas Robert, Yannick Aoustin, Eric Le Carpentier. A Simulationbased Approach to Generate Walking Gait Accelerations for Pedestrian Navigation Solutions. 9th International Conference on Indoor Positioning and Indoor Navigation (IPIN 2018), Sep 2018, Nantes, France. pp.24-27, 10.1109/IPIN.2018.8533858 . hal-01997361

\section{HAL Id: hal-01997361 \\ https://hal.science/hal-01997361}

Submitted on 14 Feb 2019

HAL is a multi-disciplinary open access archive for the deposit and dissemination of scientific research documents, whether they are published or not. The documents may come from teaching and research institutions in France or abroad, or from public or private research centers.
L'archive ouverte pluridisciplinaire HAL, est destinée au dépôt et à la diffusion de documents scientifiques de niveau recherche, publiés ou non, émanant des établissements d'enseignement et de recherche français ou étrangers, des laboratoires publics ou privés. 


\title{
A Simulation-based Approach to Generate Walking Gait Accelerations for Pedestrian Navigation Solutions
}

\author{
Mahdi Abid ${ }^{1,3}$, Valerie Renaudin ${ }^{1}$, Thomas Robert ${ }^{2,3}$, Yannick Aoustin ${ }^{4}$, Eric Le Carpentier ${ }^{4}$ \\ ${ }^{1}$ IFSTTAR, GEOLOC Laboratory, Route de Bouaye CS4, 44344 Bouguenais, France \\ ${ }^{2}$ Univ. Lyon, Université Claude Bernard Lyon 1, ${ }^{3}$ IFSTTAR, LBMC UMR_T9406, 69622 Lyon, France \\ ${ }^{4}$ LS2N, UMR 6004, École Centrale de Nantes, Université de Nantes, France \\ E-mails: \{valerie.renaudin, thomas.robert\}@ifsttar.fr, Yannick.Aoustin@univ-nantes.fr, eric.le-carpentier@ec-nantes.fr
}

\begin{abstract}
In indoor environments, pedestrian dead reckoning (PDR) is the most used strategy for pedestrian position estimation from inertial data collected with handheld devices. PDR process recursively estimates positions using step length estimation based on parametric models that take into consideration some physiological parameters, displacement features and acceleration statistical properties. The coefficients of these models need frequent adjustment to limit cumulative errors induced by alteration of gait pattern. A large experimental database providing information about human locomotion variability is required for this calibration. However, the development of such database is costly in terms of time and effort. To make the collected data as reliable as possible, several gait-affecting factors should be considered, which highly increases the number of measurement trials. In this paper, we propose an alternative way of generating locomotion data that consists in simulating human walking gait motion under different conditions. We propose a multibody system simulator taking into account possible steplevel asymmetry induced by handling a device in hand, as well as the correlation between arms and legs motions during gait. Our simulation approach was evaluated with data from overground walking experiments on one test subject. Preliminary results show some similarities between acceleration profiles related to different body parts, and the same variation trends of selected acceleration items in function of carrying mode and gait velocity.
\end{abstract}

Keywords-Handheld devices, Human walking gait, Multibody system, Inertial data, Step-level asymmetry.

\section{INTRODUCTION}

The main issue in indoor positioning is that satellite signals are weakened in indoor spaces resulting in a degradation of continuous navigation performance [1]. Pedestrian Dead Reckoning (PDR) is the most employed approach when it comes to estimate pedestrian position in such spaces. It consists on estimating the traveled distance based on inertial sensors signals [2], since they are available regardless of surrounding infrastructures. PDR recursively estimates positions using step detection, step heading and length estimation. This estimation of step length is more complex when sensors are handheld due to the complex hand motions that do not directly reflect the walking pattern, in contrary with body-fixed sensors. Parametric models that use physiological parameters (height [3]), step characteristics (frequency [4]) and acceleration signal statistical characteristics [5], either combined or independently, have been adopted for this purpose.

Most of these models assume that common gait has been performed by individuals, who are more likely to exhibit highly irregular gait motions when walking at extreme conditions e.g. under fatigue, or when handling an additional mass in hand [6]. These unpredictable irregular motions can lead into major alteration of gait pattern, which significantly affects the performance of displacement features estimation, since positioning errors at different steps are induced and accumulated over the duration of displacement. Thus, the step length models coefficients should be adjusted frequently. A large experimental database that characterizes the variability related to human locomotion parameters is needed for this calibration. In the ideal case, the collected data should provide information about the substantial variation of walking parameters across different subjects due to intrinsic body morphology and motion pattern, i.e. inter-subject variation, and about their variation induced by a single user under different walking conditions, i.e. intra-subject variation. Hence, a challenging task is to validate the models accuracy on new subjects rather than the ones these models have been adjusted on.

The development of such a database is costly in terms of time, effort and material. In effect, many available volunteers must be found in order to collect enough experimental data, and they have to provide their written consent to perform the trials. The main problems are the number of subjects that is determinant in evaluating the reliability of the empirical method, as well as subjects' distribution in terms of age, gender, and physiological characteristics (e.g. height, weight) that have to be sufficiently addressed in order to make the collected data as broadened as possible. In addition, experimental protocols should include as many gait-impacting factors as possible (e.g. physiological or sensor-induced), and these factors have to be well measured and labelled in such datasets [7]. Yet, it is hard to analyze the coexisting effects of these factors on gait patterns simultaneously. Besides, several settings of each factor should be considered in order to conduct the evaluation in a generalized and efficient way [7], which highly increases the number of measurement trials included in the dataset, and then more effort and longer acquisition time 
are needed. To overcome these issues, data collected with wide population in the wild [8], [9], where several environmental and physiological factors affect gait patterns, are used to examine the robustness of gait recognition models. However, the influence of particular factors cannot be investigated since its contributions are unlabelled and are somehow merged within such datasets.

This study intends to provide a simpler way to generate the database that could be used for calibration of pedestrian navigation models, by substituting costly experimental protocols for simulated human locomotion. We limit our study to straightforward walking scenarios. The presented human gait simulator, taking into account the correlation between upper limbs and legs movements [10], enables to predict gait motion for given displacement features and a given device carrying mode. In this paper, the model is adjusted to one test subject and assessed with overground walking experiments based on acceleration features related to different body parts.

The paper is outlined as follows. In section II, a state of the art on gait features extraction using inertial sensors is presented, as well as the walking gait features considered in this study. Section III is dedicated to the presentation of the proposed walking gait simulator. Section IV details the experimental protocol for data collection and the gait features extraction procedure. It is followed by a discussion of evaluation results of the simulation approach in Section V.

\section{WALKING GAIT FEATURES}

\section{A. State of the art on gait features extraction using inertial sensors}

The usage of inertial sensors in gait analysis is a promising method for features extraction, as they are designed to be light, cheap and portable, and to escape from the need for specific experimental environment [11]. They are non-invasive, do not alter the motion patterns [12] and can identify human activity in different environments [13], [14]. The use of 3D sensors provides more information of human motion in three planes [15]. Inertial sensors are mainly used for human motion monitoring, as well as activity recognition and advanced gait analysis [7]. Potential applications of these approaches are healthcare and disease prevention (i.e. fall detection for elderly people [16], assessment of physical activity patterns to detect gait abnormalities [11]) and sports (i.e. gait speed estimation and step count [17]). Recently, gait recognition based on inertial sensors for localization purposes has become indispensable with the appearance of smart devices and wearable systems including these sensors [7]. Gait patterns can be explicitly identified as physiological properties, i.e. 3D joint angle estimation [18], detection of gait cycle phases [13], or estimation of spatio-temporal characteristics (step length, width of walking base, gait symmetry, cadence, etc.) [19]. These patterns can be also expressed using feature extraction techniques that do not exactly provide physiological parameters but implicitly contain information on walking motion [7].

Several types of inertial sensors have been employed for gait analysis either combined, or with other non-inertial sensors
[11]. The most used sensor is accelerometer either biaxial or tri-axial. In general, accelerometers are combined with gyroscopes to decrease the error induced by accelerometer vibration and to constitute an Inertial Measurement Unit (IMU) system [19] or a self-developed multi-sensors system [13]. Magnetometers are also included in IMU systems [12]. When using more inertial sensors, the accuracy of features estimation is improved thanks to data redundancy and data fusion algorithms [20]. Nevertheless, a compromise between precision and portability should be considered.

Researchers place these sensors in different parts of the body, e.g. attached to lower limbs (foot, thigh) to accurately detect gait phases and displacement features, to upper body (waist, abdomen) or to the arms [11]. The highest locations are ears and head [21]. The acceleration of the upper parts can better assess the body stability and balance during gait [22], and contribute to a more sophisticated gait analysis [11].

\section{B. Selected walking gait features}

Let's note that a stride is composed of two consecutive steps. In this study, there are two sets of gait features that are extracted from experimental dataset:

- The displacement features that are imposed in simulation tests:

- The gait velocity $V$;

- The stride characteristics i.e. length $(d)$ defined as the distance between two consecutive prints of the same foot, and width $(w)$ defined as the lateral distance between the midlines of both feet;

- The step-level symmetry indexes i.e Temporal Symmetry Index [6] and Spatial Symmetry Index respectively notated TSI and SSI, and defined as:

$$
\begin{aligned}
& \text { TSI }=\frac{\text { Time duration of step on the device's side }}{\text { Total time duration of the stride }} \\
& \text { SSI }=\frac{\text { Length of step on the device's side }}{\text { Stride length }}
\end{aligned}
$$

Note that stride is defined between two successive initial contacts (ICs) by same foot, and a right step is the interval between left IC and following right IC.

- The acceleration features over the gait cycle (GC) related to different body parts:

- Both feet;

- The waist whose motion reflects the center of mass (COM) kinematics.

- The hand that is carrying a mass.

The second set of features is used for the validation of the developed gait simulator.

\section{3D WALKING GAIT SIMULATOR OVER A STRIDE}

\section{A. Presentation of the $3 D$ biped equipped with handheld mass}

An anthropomorphic 3D biped with two identical threelink legs, a pelvis, a trunk, a head-neck, and two-link arms is considered (cf. Fig. 1). All segments are supposed rigid and linked by frictionless rotational joints. Denavit-Hartenberg 
convention [23] is used to express link frames situation w.r.t. a frame linked to the stance foot in function of joint angles. The joints degrees of freedom (DOF) are defined in reference to human gait analysis detailed in [24], [25]. To make the

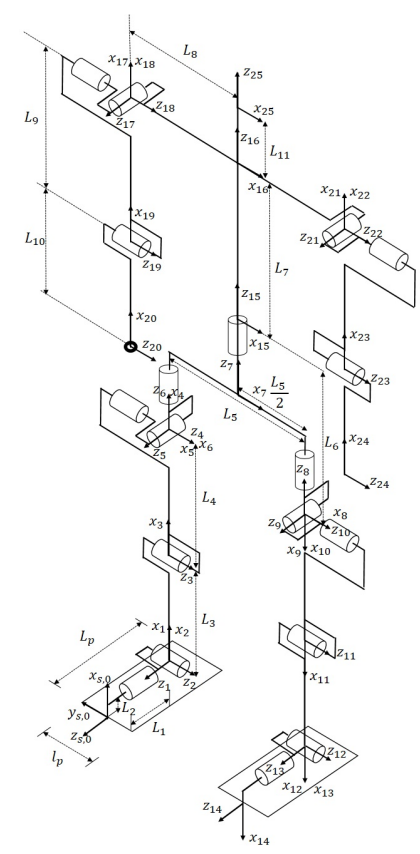

FIG. 1 -. The 19-link biped model with dimensions and local frames in accordance with Denavit-Hartenberg convention [23].

biped fit a test subject, anatomical definition found in [26] is considered to estimate the segments lengths. In addition, the regression of Dumas [26] are used for BSIPs (body segment inertial parameters) estimation. These parameters correspond for each link to its mass, COM relative position and inertias. The added mass in hand is assumed to be rigidly held, then its inertial parameters are merged with those of the forearm.

\section{B. Definition of walking gait cycle}

The GC of the biped is defined as the stride, it is composed respectively of a right single support (SS) phase (right support foot), a DS phase, a left SS phase, and a second DS phase. The DS phases are instantaneous and these assumptions are considered:

- There is only flat contact of both feet with the ground.

- There are no roll and yaw rotations of the pelvis, and the axial rotation between trunk and pelvis segments is null.

We assume joint positions continuity at the impact, then final positions of each step is determined from initial positions of the next step using an inversion matrix expressing the exchange of role of joints after the impact. However, there is a joint velocity discontinuity at the impact, and velocities after the impact are function of velocities before the impact following an impact model.

\section{Dynamic and impact models}

Using the Newton-Euler algorithm, the biped's equations of motion are obtained in the following form:

$$
\left[\begin{array}{c}
\mathbf{R}_{0} \\
\Gamma
\end{array}\right]=\operatorname{IDM}\left(\mathbf{q}, \dot{\mathbf{q}}, \ddot{\mathbf{q}}, \mathbf{R}_{14}\right)
$$

where $\mathbf{R}_{0} \in \mathbb{R}^{6}$ is the wrench of ground reaction forces on the stance foot, $\mathbf{R}_{14} \in \mathbb{R}^{6}$ is the wrench of forces exerted by the swing foot on the ground, $\Gamma \in \mathbb{R}^{19}$ is the vector of joint torques, $\mathbf{q}, \dot{\mathbf{q}}, \ddot{\mathbf{q}} \in \mathbb{R}^{19}$ are respectively the position, velocity and acceleration vectors. $\mathbf{R}_{14}=\mathbf{0}_{6 \times 1}$ in SS. The biped's generalized coordinates are expressed by $\mathbf{X}=\left(\mathbf{x}_{0} ; \alpha_{0} ; \mathbf{q}\right) \in$ $\mathbb{R}^{25}$ where $\mathbf{x}_{0}$ and $\alpha_{0}$ define respectively the position and orientation of the frame $R_{0}$ with respect to the reference frame $R_{s}$. The biped velocity is $\mathbf{V}=\left(\mathbf{v}_{0} ; \omega_{0} ; \dot{\mathbf{q}}\right) \in \mathbb{R}^{25}$ and its acceleration is $\dot{\mathbf{V}}=\left(\dot{\mathbf{v}}_{0} ; \dot{\omega}_{0} ; \ddot{\mathbf{q}}\right) \in \mathbb{R}^{25}$ where $\mathbf{v}_{0}$ and $\omega_{0}$ are the linear and angular velocities of the stance foot. The dynamic model in DS can be written:

$$
\mathbf{D}(\mathbf{X}) \dot{\mathbf{V}}+\mathbf{C}(\mathbf{V}, \mathbf{q})+\mathbf{G}(\mathbf{X})+\mathbf{D}_{\mathbf{J}}(\mathbf{q}) \mathbf{R}_{14}=\mathbf{D}_{\Gamma} \boldsymbol{\Gamma}+\mathbf{D}_{0} \mathbf{R}_{0}
$$

where $\mathbf{D} \in \mathbb{R}^{25 \times 25}$ is the inertia matrix, $\mathbf{C} \in \mathbb{R}^{25}$ is the vector of Coriolis and centrifugal forces, $\mathbf{G} \in \mathbb{R}^{25}$ is the vector of gravity, $\mathbf{D}_{\mathbf{J}}^{\top} \in \mathbb{R}^{6 \times 25}$ is the Jacobian matrix reflecting the effects of ground reaction on the landing foot, $\mathbf{D}_{\Gamma}=$ $\left(\mathbf{0}_{6 \times 19} ; \mathbf{I}_{19 \times 19}\right)$ is a matrix allowing to take into consideration the joint torques, $\mathbf{D}_{0}=\left(\mathbf{I}_{6 \times 6} ; \mathbf{0}_{19 \times 6}\right)$ is a matrix allowing to take into account the effects of ground reaction on the stance foot. The impact model can be obtained by integrating the dynamic model (4) during the impact duration which tends to zero:

$$
\mathbf{D}(\mathbf{X}(T)) \Delta \mathbf{V}=\mathbf{D}_{0} \mathbf{I}_{0}-\mathbf{D}_{\mathbf{J}} \mathbf{I}_{14}
$$

where $\mathbf{I}_{0}$ and $\mathbf{I}_{14}$ are the intensity of Dirac delta functions for the forces $\mathbf{R}_{0}$ and $\mathbf{R}_{14}, \Delta \mathbf{V}=\left(\mathbf{V}^{+}-\mathbf{V}^{-}\right)$is the variation of velocity at the impact, $\mathbf{X}(T)$ is the biped's configuration at the impact. After the impact, the previous stance foot takes off the ground and its impulsive forces are equal to zeros $\mathbf{I}_{0}=\mathbf{0}_{6 \times 1}$. The velocity of the landing foot after impact is null, then:

$$
\mathbf{D}_{\mathbf{J}}^{\top} \mathbf{V}^{+}=\mathbf{0}_{6 \times 1}
$$

Equations (5) and (6) give the impact model:

$$
\left[\begin{array}{cc}
\mathbf{D}(\mathbf{X}(T)) & \mathbf{D}_{\mathbf{J}} \\
\mathbf{D}_{\mathbf{J}}^{\top} & \mathbf{0}_{6 \times 6}
\end{array}\right]\left[\begin{array}{l}
\mathbf{V}^{+} \\
\mathbf{I}_{14}
\end{array}\right]=\left[\begin{array}{c}
\mathbf{D}(\mathbf{X}(T)) \mathbf{V}^{-} \\
\mathbf{0}_{6 \times 1}
\end{array}\right]
$$

\section{Joint motion generation}

Joint positions need to be approximated during the SS phase of the step labelled $j$ on the global time interval $\Omega=\left\{t_{0}=0, t_{N+1}=T_{j}\right\}, j=1,2$. For interpolation, we consider $N$ intermediate configurations in SS that are uniformly spaced at the time knots: $t_{k}=k . h$, where $h=\frac{T_{j}}{(N+1)}, k=1, \ldots, N$. Each model DoF $\mathbf{q}_{i}$ is approximated by a function $\phi_{i}(t)$ defined on $\Omega$ and obtained by the concatenation of $N+13^{\text {rd }}$ order polynomial functions successively defined on the intervals $\Omega_{k}=\left\{t \mid t_{k} \leq t<t_{k+1}\right\}$ :

$$
\varphi_{i, k}(t)=\sum_{l=0}^{3} a_{i, k}^{l}\left(t-t_{k}\right)^{l}, \quad k=0, \ldots, N \quad \forall t \in \Omega_{k}
$$


where $a_{j, k}^{l}$ are the polynomial coefficients. There are $N+1$ polynomials with 4 coefficients, $4(N+1)$ independent conditions are necessary to define each function $\phi_{i}(t)$ :

- Position conditions at SS and DS $(N+2$ conditions);

- Velocity conditions at DS (two conditions);

- Joint position, velocity and acceleration continuity conditions at $\mathrm{SS}$ ( $3 N$ conditions);

Note that position and velocity parameters used for interpolation are deduced from the optimization variables presented in section III-E1.

\section{E. Optimization process}

Parametric optimization algorithm based on Sequential Quadratic Programming (SQP) method [27] is used to solve the nonlinear constrained problem.

1) Optimization variables: Due to continuity and cyclicity conditions, and to the choice of a minimal set of variables defining the legs configurations at DS instants (cf. Fig. 2 ), the variables defining the motion over the GC can be significantly reduced. The optimized set comprises for each step the variables presented in Table I. The number of variables is reduced in texting mode due to the constrained arm configuration. Corresponding joint rate variables are set to zero and joint positions are fixed so that the hand has a defined position w.r.t. the head i.e. The position of the origin $O_{20}$ linked to the hand w.r.t. the origin $O_{16}$ linked to the head is ${ }^{16} \mathbf{P}_{20}=\left(\frac{-L_{8}}{2}, \frac{-2 L_{10}}{3}, \frac{-L_{7}}{2}\right)_{R_{16}}$, where $L_{7}, L_{8}$, and $L_{10}$ are respectively the trunk length, the lateral distance between the head and shoulder joint center, and forearm length.

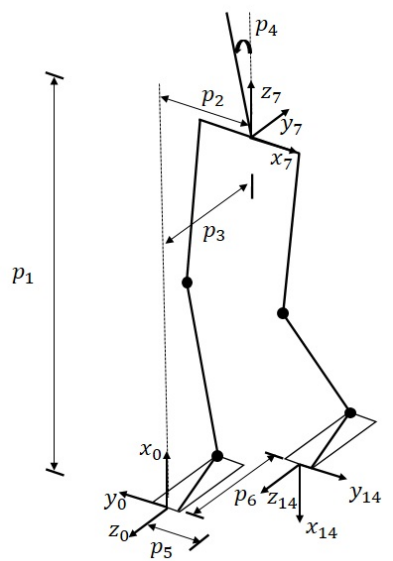

FIG. 2 -. The six variables determining the locomotor system configuration at DS: four variables for pelvis situation w.r.t. the stance foot, and two parameters for step length and width.

2) Cost functional: The gait energy dissipated over the GC is minimized in this process. Then we define a sthenic criterion for each step labeled $j$, of period $T_{j}$ and length $d_{j}$, based on joint torques values [28]:

$$
C_{\Gamma, j}=\frac{1}{d_{j}} \int_{0}^{T_{j}} \boldsymbol{\Gamma}_{j}(t)^{\top} \boldsymbol{\Gamma}_{j}(t) \mathrm{d} t
$$

TAB. I -

Optimization variables corresponding to the step labelled $j, j=1,2$ and to $N$ intermediate time knots. (S): Swinging mode, (T): Texting mode.

\begin{tabular}{l|cc} 
Set of variables & \multicolumn{2}{|c}{ Number of variables } \\
& $(\mathrm{S})$ & $(\mathrm{T})$ \\
\hline $\mathbf{q}_{j}\left(t_{i, j}\right), i=1, \ldots, N$ : Intermediate configurations & $19 N$ & $16 N$ \\
$\dot{\mathbf{q}}_{j}\left(T_{j}\right):$ Joint velocities just before the impact & 19 & 16 \\
$\left(p_{1, j}, p_{2, j}, p_{3, j}\right):$ Hips center position in initial DS & 3 & 3 \\
$p_{4, j}:$ Initial pelvis orientation in the sagittal plane & 1 & 1 \\
$\mathbf{q}_{j, \text { arm }}(t=0):$ Arms configuration in initial DS & 6 & 3
\end{tabular}

where $\boldsymbol{\Gamma}_{j}$ is the vector of joint torques during the step $j$. The energetic cost function of the stride $C_{\Gamma}$ can be expressed in function of both steps criteria $C_{\Gamma, 1}$ and $C_{\Gamma, 2}$ as follows:

$$
C_{\Gamma}=\mathrm{SSI} \cdot C_{\Gamma, 1}+(1-\mathrm{SSI}) \cdot C_{\Gamma, 2}
$$

Another performance criterion quantifies the trunk's deviation from upright position [29] over the stride's duration:

$$
C_{U T P}=\int_{0}^{T}\left(\mathbf{P}_{O_{16}}(t)-\mathbf{P}_{O_{7}}(t)\right) \cdot \boldsymbol{g} \mathrm{d} t
$$

where $\boldsymbol{P}_{O_{16}}$ is the cartesian position of the trunk's upper extremity, $\boldsymbol{P}_{7}$ is the cartesian position of the hips middle point, and $\boldsymbol{g}$ is the gravity vector. Consequently, the cost functional for a stride is given by:

$$
C_{f}=C_{\Gamma}+\rho \cdot C_{U T P}
$$

where $\rho>0$ is a penalty factor.

3) Constraints: To obtain human-like walking patterns, the following constraints are imposed:

- Each joint must not exceed its biomechanical limits on position, velocity and torque;

- In SS, constraints on the swing foot position are imposed in order to avoid collision with the ground or with the support leg;

- To satisfy unilateral contact between the support foot and the ground, Zero Moment Point (ZMP) is constrained within the support polygon defined by the four corners of the stance foot, during SS phases;

- Ground reaction of the stance foot in SS and the impact forces of the landing foot at instantaneous DS are constrained inside a friction cone of a coefficient $\mu$ to avoid slipping, and must be directed upward to avoid take-off;

- The inertial and gravity force (IGF) moment about the ZMP is given by:

$\mathbf{Y}^{I G F}=\sum_{i=1}^{n_{s}}\left(\mathbf{p}_{\mathrm{COM}}^{i}-\mathbf{p}_{\mathrm{ZMP}}\right) \times m_{i}\left(\mathbf{g}-\mathbf{a}_{\mathrm{COM}}^{i}\right)$

where $\mathbf{p}_{\mathrm{ZMP}}$ is the ZMP position, $\mathbf{p}_{\mathrm{COM}}^{i}$ and $\mathbf{a}_{\mathrm{COM}}^{i}$ are the $i^{\text {th }}$ segment's COM position and acceleration, respectively, $\mathbf{g}$ is the gravity vector, $m_{i}$ is the $i^{\text {th }}$ segment's mass, $n_{s}$ is the number of body segments.

In order to generate contralateral swing of the arms, the Zero Yawing Moment (ZYM) constraint [29] keeps the resultant yawing component (in the vertical $(x)$ direction) 
under a small upper bound of magnitude $(=0.1$ N.m in this study).

- For texting mode, additional constraint keeps the same distance between the carried mass and the head.

\section{EXPERIMENTATIONS}

\section{A. Experimental setup}

1) Equipment: Two ULISS devices [30] and two PERSY systems [31] designed by IFSTTAR-GEOLOC Laboratory are used for data collection. Each ULISS device contains a nineDOF inertial and magnetic unit, a high sensitivity GNSS receiver, a battery, and a memory card. It weights $129 \mathrm{~g}$ which is approximately the mass of a smartphone. Raw inertial data are collected at $200 \mathrm{~Hz}$ frequency. During trials, one ULISS device is held in hand by the subject while the other one is attached to the rear of the belt (cf. Fig. 3).

PERSY systems has been designed for reference positioning in indoor environments. For this task, they must be attached to both feet (cf. Fig. 3). They are lightweight, wireless, and comprise an internal logging system and a battery. To obtain the best possible position using MEMS technology, three inertial and GNSS sensors running at $160 \mathrm{~Hz}$ are embedded in each PERSY: a high precision IMU STIM300 with a gyro ranging up to $800 \%$, a magnetometer HMC5983, and an M8T GNSS receiver. All devices have GNSS running at $5 \mathrm{~Hz}$ and autonomy of four hours. All data are timestamped using GPS time.

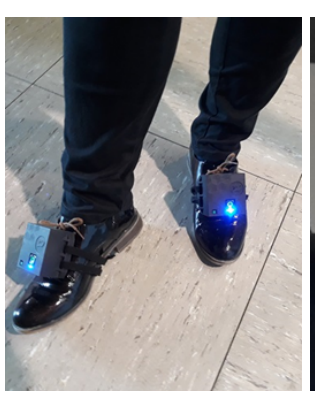

(a)

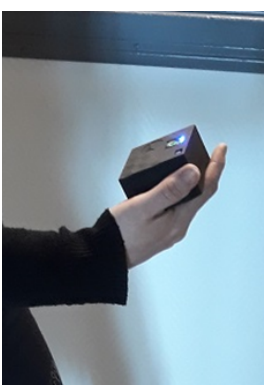

(b)

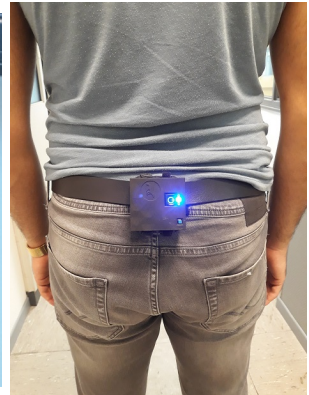

(c)
FIG. 3 -. Placement of different devices during walking trials: (a) PERSYs; (b) ULISS 1; (c) ULISS 2.

2) Environment: Experiments took place at the site of IFSTTAR institute in Bouguenais, France. Walking tests were performed indoor in a 55m-long hall, in one of the site's buildings. Before each acquisition, magnetometers calibration in outdoor is needed, as well as a static phase of about one minute to get GPS time for data synchronization. Data collection lasts about 30 minutes.

\section{B. Subject and scenarios}

One healthy 26-year old volunteer participated in data collection. The subject's height is $1.6 \mathrm{~m}$, and he weighs 58 $\mathrm{kg}$.

The following device carrying modes were tested for straight walking scenarios (cf. Fig. 4):

- Swinging mode (S1): the subject is walking with freely swinging arms, and handling ULISS 1 in the right hand;

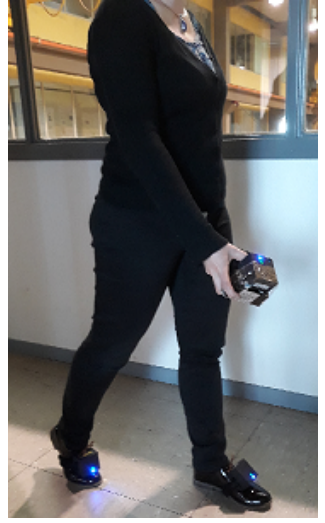

(a)

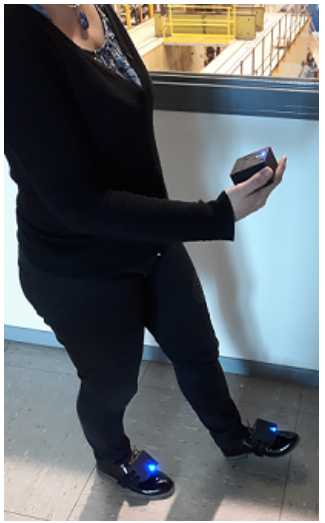

(b)
FIG. 4 -. Tested device carrying modes: (a) Swinging; (b) Texting.

- Texting mode (T): the subject is asked to walk while watching the device in hand so that right arm configuration is comfortable for texting or reading;

- Swinging mode (S2): the same as (S1), however an additional mass is taped to ULISS 1 (cf. Fig. 5) and the total handled mass is $627 \mathrm{~g}$;

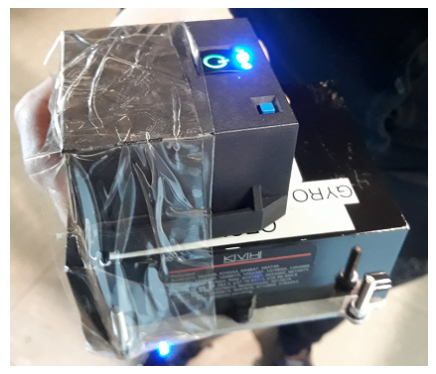

FIG. 5 -. The additional mass taped to ULISS device.

Each walking scenario was performed at three walking speeds: comfortable $\left(V_{2}\right)$, slow $\left(V_{1}\right)$ and fast $\left(V_{3}\right)$ speeds. These different speeds were self-selected by the participant relative to his comfortable pace. To avoid any order effects, the subject was free to select speeds order and the order of carrying modes within the same gait speed. For each speed/carrying mode combination, the participant walked twice along the hall, back and forth between the start and the end of the walkway. The trials were separated by static phases of $10 \mathrm{~s}$. The subject was instructed to maintain the same speed and a straightforward direction during each trial.

\section{Experimental extraction of selected gait features}

For each walking condition, selected gait features were calculated for 25 consecutive strides centered in trials time interval i.e. out of acceleration/deceleration phases. Since there are two walks by condition, i.e. back and forth, gait features are averaged over 50 strides.

1) Extraction of displacement features: To extract the characteristics of each stride, step events need to be detected for both feet. For this purpose, ZVD (Zero velocity detection) method [32] was applied to PERSYs specific force in the 
navigation frame NED (North-East-Down). Specific force is projected in NED using MAGYQ algorithm [33] that estimates attitude angles of the IMU in the navigation frame. ZVD method calculates variance on a sliding window on acceleration signal, then compares it to a threshold to get the period when the foot is stationary. The detected step events correspond, for each foot, to the end of flat foot phase. The variance threshold and the width of the sliding window are adapted so that there is no under/over detection of steps. The sliding window is wider for lower gait speeds, and the variance threshold increases for higher gait velocities. Note that the same values of width and threshold are used for a given subject/gait speed combination, regardless the carrying mode.

Once steps are detected, average temporal characteristics were determined for each walking condition. Step instants were used to calculate strides durations. Since there was no difference in mean values between left and right steps, stride duration was calculated in terms of the mean values for both feet. Relative durations between right and left steps detections were used to calculate TSI indexes.

A developed software was used to calculate reference trajectories of both feet w.r.t. a base station located in the test site, based on data collected with each PERSY. Novel data fusion algorithms are used to obtain trajectories with drift errors less than $0.5 \%$, based on results found in [31]. These trajectories allow to obtain footprints coordinates corresponding to step instants. Stride length was calculated from distances between each two consecutive footprints, averaged on both feet. Then, gait velocity was determined from mean stride length and duration. Using relative positions of right footprints w.r.t. to left footprints, as well as walking direction proper to each stride, step width values and SSI indexes were calculated.

2) Extraction of acceleration profiles: Due to the cyclostationary nature of steady gait, a segmentation of inertial data is conducted in order to calculate averaged patterns that can be compared with profiles generated by simulation. Acceleration signals collected from different devices can be synchronized and divided into GCs. One GC lasts from the beginning of left rotation subphase about the toe until the end of next flat foot phase of the same foot (cf. Fig. 6). GCs time intervals are then defined between successive step events of the left foot. Perturbations caused by small variations in gait

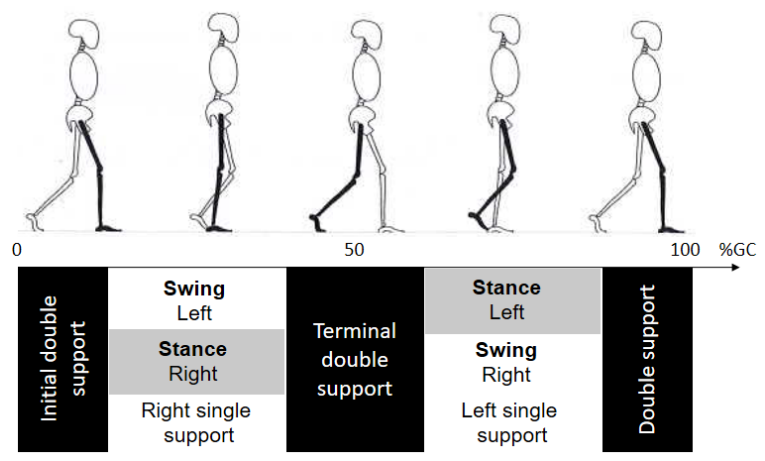

FIG. 6 -. The subdivisions of GC [24]. velocity can result in temporal variations in walking patterns. First, the lengths of extracted GCs are normalized to the mean length, while preserving the same shape of each signal pattern. Spline interpolation is used for this normalization. Second, an optimal matching of stride-specific profiles is needed since alignment impacts the temporal shape of the averaged pattern. Progressive cycle alignment is performed by dynamic time warping (DTW) approach [34] so that amplitude attenuation and phase shift are avoided for aligned signals. Then, acceleration signal pattern is obtained by averaging the aligned profiles.

\section{RESULTS}

Preliminary results show a difference in magnitude between signals from experiments and simulation due to the simplifying assumptions considered in the model, then a qualitative evaluation of our simulation results is conducted. This evaluation includes comparison with experimental data based on normalized acceleration patterns corresponding to the speed/carrying mode combination $V_{2} /(\mathrm{S} 1)$, as well as variation tendencies of some acceleration items across walking scenarios. Normalized signals are obtained by dividing each signal by its maximum value throughout the GC.

\section{A. $3 D$ acceleration of the feet}
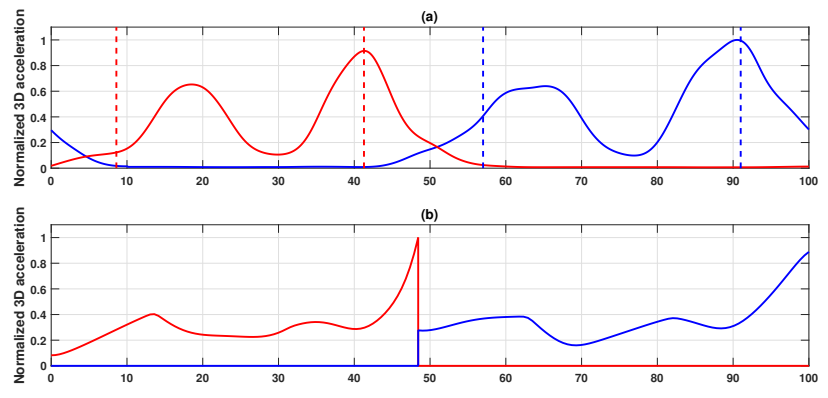

FIG. 7 -. 3D acceleration profile of the right (blue) and left (red) foot during a $G C$ for the walking condition $V_{2} /(S 1)$ : (a) experimental data, (b) simulation data. The dashed lines indicate SS phases.

Fig. 7 shows 3D acceleration profiles of both feet for experimentation and simulation. For both cases, two main peaks of acceleration are observed in SS: the first occurs at initial swing (about $30 \%$ of SS) serving as a base for swinging leg advancement, and the second occurs at the landing foot IC with the ground which marks the end of SS. For simulation, there is an abrupt transition from peak acceleration to zero at the impact instant because the DS is considered instantaneous. In contrast, this transition is smooth in experimental signal due to heel rotation of the front foot in DS. A smaller acceleration peak is observed in simulation (around 70\% of SS) as a result of foot deceleration for preparation of flat foot contact with the ground.

For both simulation and experiments, peak values of acceleration profiles significantly increase with the gait velocity, and within the same velocity, there is no significant impact of carrying mode on these values. 

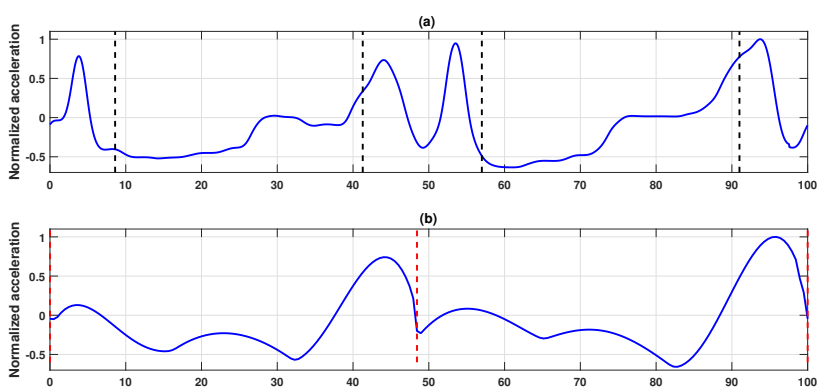

FIG. 8 -. Vertical acceleration profile of the COM during a GC for the walking condition $V_{2} /(S 1)$ : (a) experimental data, (b) simulation data. The black dashed lines indicate DS phases, and red dashed lines indicate impact instants.
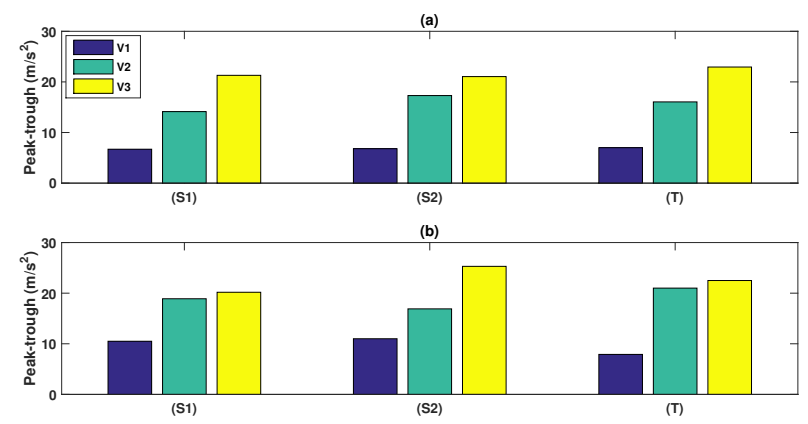

FIG. 9 -. The peak-trough differences of COM vertical acceleration for different walking conditions: (a) experimental data, (b) simulation data.

\section{B. COM's vertical acceleration}

Similar shapes of COM's vertical acceleration profiles are obtained for simulation and experimental cases (cf. Fig. 8). In experiments, two peaks occur in each DS corresponding to front foot heel rotation and rear foot rotation about the toe. For simulation, peaks occur at initial and final subphases of SS. Differences in peaks values are mainly due to the difference between joint velocities and accelerations before and after the instantaneous impact. In both experimentation and simulation, the negative peak amplitude is about half of the positive one.

Fig. 9 shows the differences between peak and trough values of COM vertical acceleration for different walking conditions. Our simulator provides the same variation trend in terms of gait velocity as in experimentation. Within the same device carrying mode, the peak-trough difference significantly increases with walking speed. For a given walking velocity, differences in vertical acceleration items are mainly due to differences between step length values.

\section{Hand's $3 D$ acceleration}

For swinging mode scenarios, experimental data show that peaks of 3D acceleration of the hand occur in DS phases (cf. Fig. 10). In simulation, maximum values of $3 \mathrm{D}$ acceleration are obtained just after the impact instants. In both experiments and simulation, minimum acceleration values occur in SS phases during arm swing motion.
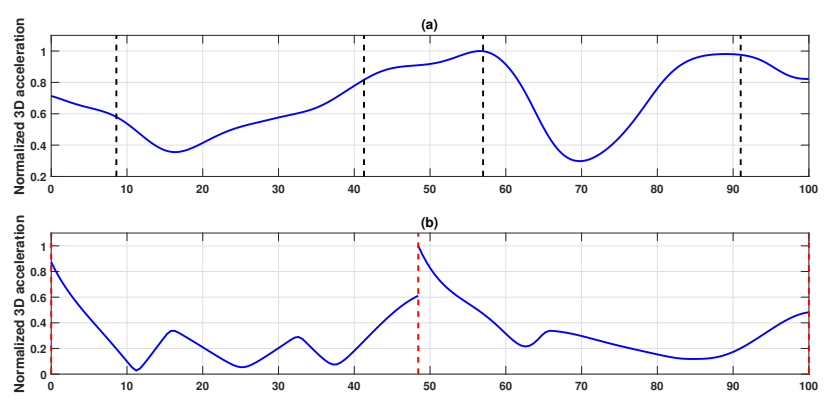

FIG. 10 -. $3 D$ acceleration profile of the hand during a GC for the walking condition $V_{2} /(S 1)$ : (a) experimental data, (b) simulation data. The black dashed lines indicate DS phases, and red dashed lines indicate impact instants.
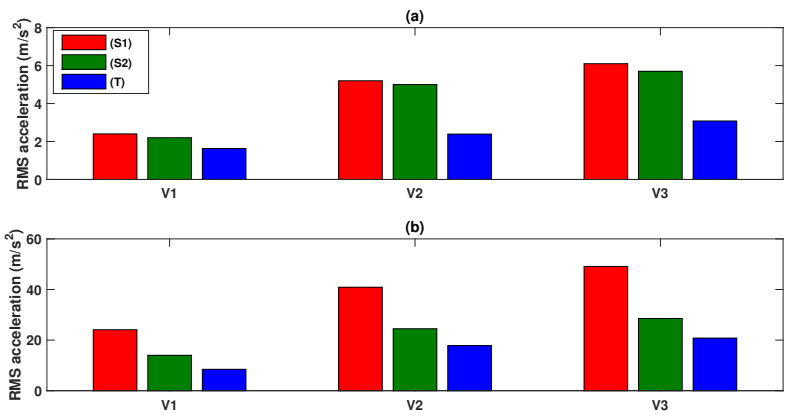

FIG. 11 -. RMS values of hand's $3 D$ acceleration for different walking conditions: (a) experimental data, (b) simulation data.

Root mean square (RMS) values of hand's 3D acceleration for different walking scenarios are shown in Fig. 11. A slight decrease of the RMS value is observed for the mode (S2) compared to (S1) because of the additional mass carried by the hand. This difference in RMS value is more remarkable in simulation data since the additional mass considerably decreases the optimal swing magnitude of the loaded arm during GC. In addition, both data show a significant decrease of RMS levels for the mode (T) w.r.t. swinging modes as a result of the constrained arm configuration. Both results show that, within the same carrying mode, RMS value increases in function of the gait velocity.

\section{CONCLUSION AND FUTURE WORK}

For indoor positioning, PDR is the most used strategy when it comes to estimate pedestrian position from inertial data sensed by handheld devices. In PDR process, step length is estimated using parametric models taking into account some physiological parameters of the user, displacement features and acceleration statistical properties. These models are suitable for the common human gait, and alterations of gait pattern induced by irregular motions lead to increased positioning error at each step. Consequently, frequent adjustment of step length models coefficients is required. This calibration needs large experimental database that characterizes inter/intrasubject variation of human gait parameters. The collection of such a database is costly in terms of time and effort since it should include as many gait-impacting factors as 
possible, which highly increase the number of measurement trials. In this paper, we aim to introduce a human gait simulator based on a multibody system in order to provide a simpler way to generate human locomotion database. To evaluate our simulator, simulation outputs were compared with overground walking data collected with one test subject. Despite the simplifying assumptions considered in simulation, preliminary results showed similarities between acceleration profiles related to different body parts. Furthermore, the same variation tendencies of some acceleration items as function of carrying mode and gait velocity are observed for both data. Based on these results, our simulator stands useful for finding correlations between acceleration signals and displacement characteristics, and more accurate calibration of step length models is foreseen.

Future work will mainly be dedicated to introducing DS phases in GC definition in order to obtain shapes and amplitudes of acceleration profiles closer to those of real walking. Validation with experimental data of several subjects is also targeted in order to qualify the model's capability of coping with gait pattern variability.

\section{REFERENCES}

[1] J. H. Ryu, G. Gankhuyag, and K. T. Chong, "Navigation system heading and position accuracy improvement through GPS and INS data fusion," Journal of Sensors, vol. 2016, 6 pages, 2016.

[2] A. Mikov, A. Moschevikin, A. Fedorov, and A. Sikora, "A localization system using inertial measurement units from wireless commercial handheld devices," in International Conference on Indoor Positioning and Indoor Navigation, Oct 2013, pp. 1-7.

[3] V. Renaudin, M. Susi, and G. Lachapelle, "Step length estimation using handheld inertial sensors," Sensors, vol. 12, no. 7, pp. 8507-8525, 2012.

[4] S. Yang and Q. Li, "Ambulatory walking speed estimation under different step lengths and frequencies," in 2010 IEEE/ASME International Conference on Advanced Intelligent Mechatronics, July 2010, pp. 658-663.

[5] H. Xing, J. Li, B. Hou, Y. Zhang, and M. Guo, "Pedestrian stride length estimation from IMU measurements and ANN based algorithm," Journal of Sensors, vol. 2017, 10 pages, 2017.

[6] M. Abid, V. Renaudin, Y. Aoustin, E. L. Carpentier, and T. Robert, "Walking gait step length asymmetry induced by handheld device," IEEE Transactions on Neural Systems and Rehabilitation Engineering, vol. 25, no. 11, pp. 2075-2083, May 2017.

[7] S. Sprager and M. B. Juric, "Inertial sensor-based gait recognition: A review," Sensors, vol. 15, no. 9, pp. 22 089-22 127, 2015.

[8] J. Frank, S. Mannor, J. Pineau, and D. Precup, "Time series analysis using geometric template matching," IEEE Trans. Pattern Anal. Mach. Intell., vol. 35, no. 3, pp. 740-754, March 2013.

[9] Y. Ren, Y. Chen, M. C. Chuah, and J. Yang, "User verification leveraging gait recognition for smartphone enabled mobile healthcare systems," IEEE Transactions on Mobile Computing, vol. 14, no. 9, pp. 1961-1974, Sept. 2015.

[10] S. H. Collins, P. G. Adamczyk, and A. D. Kuo, "Dynamic arm swinging in human walking," Proceedings of the Royal Society B: Biological Sciences, vol. 276, no. 1673, pp. 3679-3688, 2009.

[11] B. Zhang, S. Jiang, D. Wei, M. Marschollek, and W. Zhang, "State of the art in gait analysis using wearable sensors for healthcare applications," in 2012 IEEE/ACIS 11th International Conference on Computer and Information Science, May 2012, pp. 213-218.

[12] N. F. Ribeiro and C. P. Santos, "Inertial measurement units: A brief state of the art on gait analysis," in 2017 IEEE 5th Portuguese Meeting on Bioengineering (ENBENG), Feb 2017, pp. 1-4.

[13] T. Liu, Y. Inoue, and K. Shibata, "Development of a wearable sensor system for quantitative gait analysis," Measurement, vol. 42, no. 7, pp. 978-988, 2009.

[14] S. Tadano, R. Takeda, and H. Miyagawa, "Three dimensional gait analysis using wearable acceleration and gyro sensors based on quaternion calculations," Sensors, vol. 13, no. 7, pp. 9321-9343, 2013.
[15] S. Boerema, L. van Velsen, L. Schaake, T. Tönis, and H. Hermens, "Optimal sensor placement for measuring physical activity with a 3D accelerometer," Sensors, vol. 14, no. 2, pp. 3188-3206, 2014.

[16] F. Sposaro and G. Tyson, "ifall: An android application for fall monitoring and response," in 2009 Annual International Conference of the IEEE Engineering in Medicine and Biology Society, Sept 2009, pp. 6119-6122.

[17] S. Yang and Q. Li, "Inertial sensor-based methods in walking speed estimation: A systematic review," Sensors, vol. 12, no. 5, pp. 6102 6116, 2012.

[18] Q. Li and J.-T. Zhang, "Post-trial anatomical frame alignment procedure for comparison of $3 \mathrm{~d}$ joint angle measurement from magnetic/inertial measurement units and camera-based systems," vol. 35, no. 11, pp. 2255-2268, 2014.

[19] A. M. Sabatini, C. Martelloni, S. Scapellato, and F. Cavallo, "Assessment of walking features from foot inertial sensing," IEEE Transactions on Biomedical Engineering, vol. 52, no. 3, pp. 486-494, March 2005.

[20] T. Liu, Y. Inoue, and K. Shibata, "A wearable force plate system designed using small triaxial force sensors and inertial sensors," Adv. in Biomedical Sensing, LNEE 55, pp. 61-73, 2010.

[21] H. Menz, S. Lord, and R. C Fitzpatrick, "Acceleration patterns of the head and pelvis when walking on level and irregular surfaces," Gait and Posture, vol. 18, pp. 35-46, Sept 2003.

[22] H. Ghasemzadeh, R. Jafari, and B. Prabhakaran, "A body sensor network with electromyogram and inertial sensors: Multimodal interpretation of muscular activities," IEEE Transactions on Information Technology in Biomedicine, vol. 14, no. 2, pp. 198-206, March 2010.

[23] E. Dombre and W. Khalil, Robot Manipulators: Modeling, Performance Analysis and Control, ser. ISTE. Wiley, 2013. [Online]. Available: https://books.google.fr/books?id=x3e8GK-Z-OcC

[24] J. Perry and J. M. Burnfield, Gait Analysis: Normal and Pathological Function. SLACK Inc., 2010.

[25] J. Rose and J. Gamble, Human Walking, ser. LWW medical book collection. Lippincott Williams \& Wilkins, 2006.

[26] R. Dumas, L. Chèze, and J.-P. Verriest, "Adjustments to mcconville et al. and young et al. body segment inertial parameters," Journal of Biomechanics, vol. 40, no. 3, pp. 543-553, 2007.

[27] M. J. D. Powell, Variable Metric Methods for Constrained Optimization, Lecture Notes in Mathematics. Springer, Berlin, 1977.

[28] C. Chevallereau and Y. Aoustin, "Optimal reference trajectories for walking and running of a biped robot," Robotica, vol. 19, no. 5, pp. 557-569, August 2001.

[29] H. J. Kim, Q. Wang, S. Rahmatalla, C. C. Swan, J. S. Arora, K. Abdel-Malek, and J. G. Assouline, "Dynamic motion planning of 3D human locomotion using gradient-based optimization." Journal of biomechanical engineering, vol. 130, no. 3, p. 031002, 2008.

[30] M. Ortiz, M. De Sousa, and V. Renaudin, "A new PDR navigation device for challenging urban environments," vol. 2017, pp. 1-11, 2017.

[31] J. L. Scornec, M. Ortiz, and V. Renaudin, "Foot-mounted pedestrian navigation reference with tightly coupled gnss carrier phases, inertial and magnetic data," in 2017 International Conference on Indoor Positioning and Indoor Navigation (IPIN), Sept 2017, pp. 1-8.

[32] I. Skog, P. Handel, J. O. Nilsson, and J. Rantakokko, "Zero-velocity detection - an algorithm evaluation," IEEE Transactions on Biomedical Engineering, vol. 57, no. 11, pp. 2657-2666, Nov 2010.

[33] V. Renaudin and C. Combettes, "Magnetic, acceleration fields and gyroscope quaternion (MAGYQ)-based attitude estimation with smartphone sensors for indoor pedestrian navigation," Sensors, vol. 14, no. 12, pp. $22864-22890,2014$.

[34] H. Skutkova, M. Vitek, K. Sedlar, and I. Provaznik, "Progressive alignment of genomic signals by multiple dynamic time warping," Journal of Theoretical Biology, vol. 385, pp. 20 - 30, 2015. 